\title{
Prevalence of Sigmoid Sinus Dehiscence and Diverticulum among Adults with Skull Base Cephaloceles
}

\author{
(D) H. Sotoudeh, (D) Elsayed, (D). Ghandili, (D). Shafaat, (D).D. Bernstock, (D) G. Chagoya, (D). Atchley, \\ (D) P. Talati, (DD. Segar, (D)S. Gupta, and (D) A. Singhal
}

\begin{abstract}
BACKGROUND AND PURPOSE: Cephaloceles are relatively rare conditions caused by a congenital and/or acquired skull defect. The incidence of associated venous brain anomalies with regard to cephaloceles remains to be fully elucidated. Accordingly, we sought to assess the prevalence of sigmoid sinus dehiscence and diverticula in patients with spontaneous skull base cephaloceles.
\end{abstract}

MATERIALS AND METHODS: Our institutional data base was retrospectively queried from 2005 to 2018. Patients in whom spontaneous skull base cephaloceles were identified were ultimately included in the study cohort. These patients subsequently had their sigmoid sinuses re-evaluated with focused attention on the possible presence of dehiscence and/or diverticula.

RESULTS: We identified 56 patients: 12 men and 44 women. After re-evaluation of the sigmoid sinuses, evidence of dehiscence and/or diverticula was noted in 21 patients. The right sigmoid sinus was involved in 11 patients, and the left sigmoid sinus was involved in 7 patients, including 3 cases of diverticulum. In 3 patients, evidence of bilateral sigmoid sinus dehiscence and diverticula was noted. Female sex was associated with sigmoid sinus dehiscence and diverticula by univariate analysis $(P=.019)$. By linear regression, cephalocele volume was negatively associated with sigmoid sinus dehiscence and diverticula (coefficient, $-2266, P$ value $<.007$, adjusted $R^{2}=0.1077$ ). By univariate logistic regression using average cephalocele volume as a cutoff, we demonstrate a statistically significant finding of lower volumes being associated with sigmoid sinus dehiscence and diverticula with an odds ratio of $3.58(P=.05)$.

CONCLUSIONS: The prevalence of sigmoid sinus dehiscence and diverticula in patients with cephalocele is high. Female sex is associated with sigmoid sinus dehiscence and diverticula. The cephalocele volume appears to be inversely proportional to sigmoid sinus dehiscence and diverticula.

ABBREVIATIONS: ICP = intracranial pressure; $\mathrm{IIH}=$ idiopathic intracranial hypertension; SSDD = sigmoid sinus dehiscence and diverticula; $C S F=$ cerebrospinal fluid

A cephalocele consists of herniation of the brain (encephalocele), meninges (meningocele), and/or both (meningoencephalocele) through a skull defect. Skull base cephaloceles can be congenital, spontaneous, or secondary to a litany of causes. ${ }^{1-3}$ Congenital skull base cephaloceles are rare and constitute $<10 \%$

Received January 4, 2020; accepted after revision April 24

From the Division of Neuroradiology, Departments of Radiology (H.S., A.S.) and Neurosurgery (G.E., G.C., T.A.), University of Alabama at Birmingham, Birmingham, Alabama; Department of Radiology (S. Ghandili), Aventura Hospital, Miami, Florida; Department of Radiology and Interventional Neuroradiology (O.S.), Isfahan University of Medical Sciences, Isfahan, Iran; Russell H. Morgan Department of Radiology and Radiological Science (O.S.), Johns Hopkins University School of Medicine, Baltimore, Maryland; Department of Neurological Surgery (J.D.B., D.S. S. Gupta), Brigham and Women's Hospital, Harvard Medical School, Boston, Massachusetts; and Department of Neurological Surgery (P.T.), Massachusetts General Hospital, Harvard Medical School, Boston, Massachusetts.

H. Sotoudeh and G. Elsayed contributed equally to this work.

Please address correspondence to Houman Sotoudeh, MD, Division of Neuroradiology, Department of Radiology, University of Alabama at Birmingham, 619 19th St S, Birmingham, AL 35294; e-mail: hsotoudeh@uabmc.edu; @Houmansotoudeh

http://dx.doi.org/10.3174/ajnr.A6602 of all cephaloceles and are typically due to defects in primary ossification within the anterior neuropore but can also be due to increased intracranial pressure (ICP) in utero. ${ }^{1,4}$ Spontaneous skull base cephaloceles form most cephaloceles diagnosed in adulthood and unlike congenital cephaloceles are not associated with other gross anomalies. The osseous defects are presumed to be secondary to the pulsation of arachnoid granulations, which can be associated with increased ICP. ${ }^{1,2,5}$ The most common locations for spontaneous skull base cephaloceles are the cribriform plate, sphenoid sinus, and tegmen tympani; ${ }^{1,6,7}$ these spontaneous cephaloceles are typically asymptomatic unless a CSF leak develops. As mentioned above, secondary cephaloceles result from myriad underlying etiologies (eg, trauma, surgery, skull base neoplasms, infection, inflammation, and radionecrosis). ${ }^{1,8,9}$

Sigmoid sinus dehiscence and diverticula (SSDD) are a clinical entity that has been reported to be associated with pulsatile tinnitus. ${ }^{10,11}$ In a retrospective study by Schoeff et al, ${ }^{12}$ in 2014, 
the prevalence of SSDD in patients with pulsatile tinnitus was found to be $23 \%$ and was female-predominate. Most interesting, the prevalence of SSDD in asymptomatic patients in whom imaging was performed has been reported to be $1.2 \%$. Of note, several interventions have been described that treat the pulsatile tinnitus caused by SSDD. ${ }^{13,14}$

SSDD has been associated with other radiographic findings, including empty sella, dehiscence of the superior semicircular canal, and increased ICPs. ${ }^{12}$ Several mechanisms have been proposed for SSDD, which include pulsation of blood flow from the sigmoid sinus, osteoporosis, and the pulsation of CSF secondary to idiopathic intracranial hypertension (IIH). ${ }^{11-13,15,16}$ Of note, recent studies have failed to show an association between SSDD and pulsatile tinnitus in patients with IIH. ${ }^{16}$ It is possible that the SSDD is not the actual cause of pulsatile tinnitus and the SSDD and pulsatile tinnitus both are different manifestations of IIH.

There remain no well-established diagnostic criteria for SSDD, and diagnosis is based on visual assessment of CT scans using the bone window. Sigmoid sinus dehiscence is defined as the absence of normal cortical bone overlying the sigmoid sinus, thereby leading to direct contact of the sinus with mastoid air cells (air-on-sinus sign); this must be visible on 2 consecutive slices in 2 orthogonal planes. ${ }^{16}$ Sigmoid sinus diverticula have been defined as focal outpouchings of the sigmoid sinus into the adjacent mastoid air cells or the mastoid cortex.

The potential association of SSDD and skull base cephaloceles (another manifestation of IIH) is currently unknown. Understanding such a relationship may ultimately be helpful in further elucidating the governing physiopathology of SSDD; strong associations between SSDD and cephaloceles would appear to favor CSF pulsation as the cause of SSDD as opposed to venous pulsations. Accordingly, we performed a retrospective study to quantify the prevalence of SSDD in patients with known skull base cephaloceles. In addition, we also sought to describe the association between SSDD and the size, location, contents, and symptoms of the patients with cephaloceles.

\section{MATERIALS AND METHODS \\ Patients}

All experiments and methods were performed in accordance with relevant guidelines and regulations set forth by the institutional review board of the University of Alabama at Birmingham, which approved the study (IRB-300000880). The study was performed in compliance with guidelines and regulations set forth by the National Institutes of Health. All patients were included as part of the institutional review board criteria for human research. All Health Insurance Portability and Accountability Act identifiers have been deleted from the presented images. Our institutional PACS data base was queried for the word "cephalocele" in all radiology reports from 2005 to 2018. Adult patients with spontaneous skull base cephaloceles were included in this study. Available imaging for each patient included a head CT with or without contrast, brain MR imaging with or without contrast, and/or a maxillofacial/temporal bone CT. These films were reviewed by a neuroradiologist with more than a decade of clinical experience.

\section{Outcomes}

Anomalies involving the sigmoid sinuses were documented in these patients with focused attention on dehiscence and diverticula. As mentioned above, sigmoid sinus dehiscence was defined as loss of normal bone coverage of the sigmoid sinus and direct contact of the sigmoid sinus with mastoid air cells (air-on-sinus sign) in at least 2 consecutive slices in 2 orthogonal planes. Sigmoid sinus diverticulum was defined as a focal outpouching of the sigmoid sinus into the adjacent mastoid air cells or the mastoid cortex. Note that the presence of a sigmoid sinus diverticulum does not necessarily suggest the presence of dehiscence but rather indicates an abnormal focal outpouching of the sigmoid sinus. The radiologist also documented the location, size, and content of cephaloceles (ie, meninges versus meninges and brain tissue). The patient's symptoms were obtained from the study indication and/or a review of the medical records. Cephalocele content was defined using MR imaging (ie, CSF versus CSF with brain tissue). If MR imaging was not available, the surgical note, the density of cephalocele, or the tethering of atrophic brain tissue toward the cephalocele or both were used as a proxy for the cephalocele. If no brain tissue was seen during an operation, the density of the cephalocele was equal to that of CSF, and there was no tethering of atrophic brain tissue toward the cephalocele, the cephalocele was assumed to contain only CSF. The cephalocele content was determined via MR imaging in all except 4 patients. In these 4 patients, the cephaloceles were isodense with CSF on $\mathrm{CT}$ and no evidence of tethering of brain tissue was noted. Three of these patients underwent surgical repair, and no brain tissue was noted during the operation. Accordingly, all 4 patients were considered to have only CSF-containing cephaloceles.

\section{Statistical Analyses}

SSDD and cephalocele categoric characteristics were analyzed using $\chi^{2}$ tests, and SSDD and cephalocele continuous characteristics were analyzed using a nonparametric approach with KruskalWallis tests. Cephalocele volume was calculated by the following equation: Volume $=$ Product of Triplanar Dimensions $\times 0.5$. A single-variable linear regression was performed between volume and SSDD to help determine the correlation. A supportive singlevariable logistic regression was performed using the average volume as a cutoff for a binomial dependent variable relative to SSDD. A multivariate logistic regression, controlling for sex, laterality, location, and cephalocele volume was performed with SSDD as the dependent binomial variable. Statistical analysis based on individual locations of the cephaloceles was not possible because of the small number within each group. We, therefore, pooled the participants into 3 main categories for further analyses: 1) anterior cephaloceles (ethmoidal, sphenoidal, cribriform plate, and sellar cephaloceles), 2) foraminal cephaloceles (cephaloceles through foramen of ovale and the Meckel cave), and 3) temporal cephaloceles (temporal apex and tegmen tympani cephaloceles).

\section{RESULTS}

Fifty-six patients with cephaloceles were included in our retrospective study. Forty-four patients were women (79\%). The average age of the group was 50.9 years. An anterior cephalocele (through the ethmoid and sphenoid sinuses, cribriform plate, and 


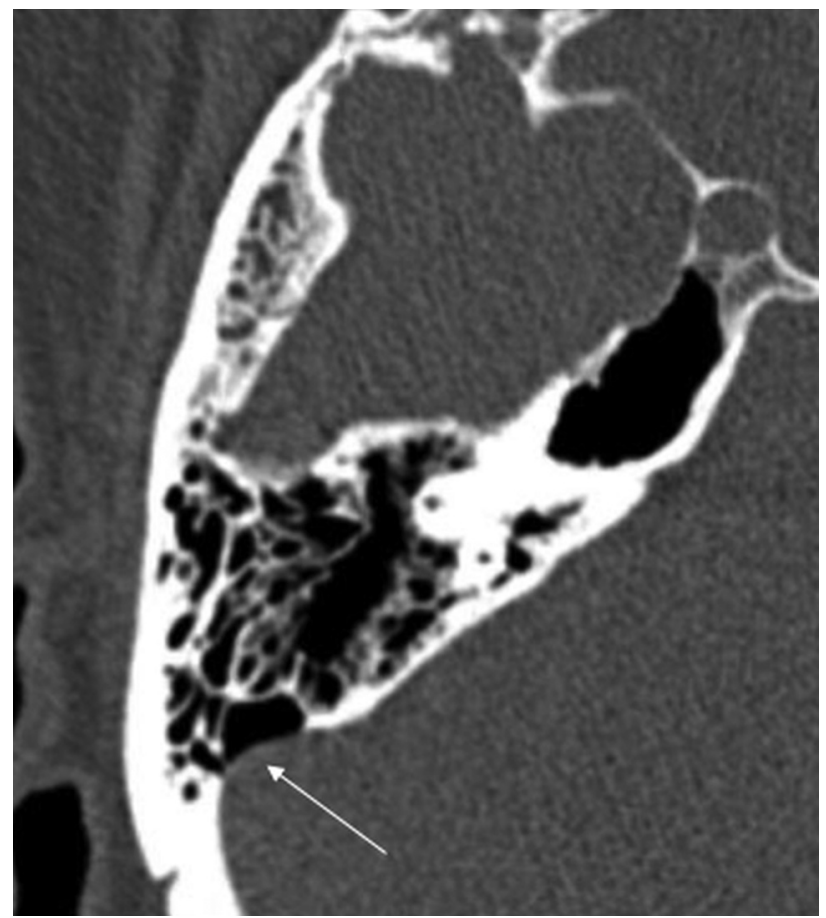

FIG 1. Dehiscence of the right sigmoid sinus (arrow). Lack of osseous coverage of the sigmoid sinus with the air-on-sinus sign.

The most common presenting symptom was rhinorrhea ( 24 patients, $42.9 \%)$. In 22 patients (39.3\%), the cephalocele was diagnosed incidentally during work-up for headaches (without other symptoms related to IIH). Other presenting symptoms were headaches secondary to $\mathrm{IIH}$ in 2 patients $(3.6 \%)$, otorrhea in 1 patient $(1.8 \%)$, bilateral hearing loss in 1 patient $(1.8 \%)$, meningitis in 4 patients $(7 \%)$, and meningitis with CSF leak in 2 patients (3.6\%). Twenty-three patients $(41.1 \%)$ were managed conservately, and 33 patients (58.9\%) underwent surgical repair.

Of 56 patients with cephaloceles, 21 patients were also shown to have SSDD, a prevalence of $37.5 \%$. Most of these patients were women, with only 1 man having been identified. In 11 patients, the right sigmoid sinus (Fig 1) was involved, and in 7 patients, the left sigmoid sinus was involved, including 3 cases of diverticulum (Fig 2). In 3 patients, evidence of bilateral SSDD was noted. The presence of an SSDD did not contribute to any group differences in the location of a cephalocele, content, or symptoms (Table 1).

Most interesting, there was a significant inverse association between the volume of the cephalocele and the presence of an SSDD. Patients with an SSDD had a lower mean cephalocele volume $\left(1562.4 \pm 1511.8 \mathrm{~mm}^{3}\right)$ compared with those without one (mean $\left.=3828.6 \pm 3559.4 \mathrm{~mm}^{3}, P=.015\right)$. By multivariate logistic regression, controlling for sex, laterality, and location, cephalocele volume remained significantly associated with SSDD $(P=.036)$. In addition, as cephalocele volume decreased, there was an association with SSDD (coefficient, $-2266 ; P$ value $<.007$, adjusted $R^{2}=0.1077$ ).

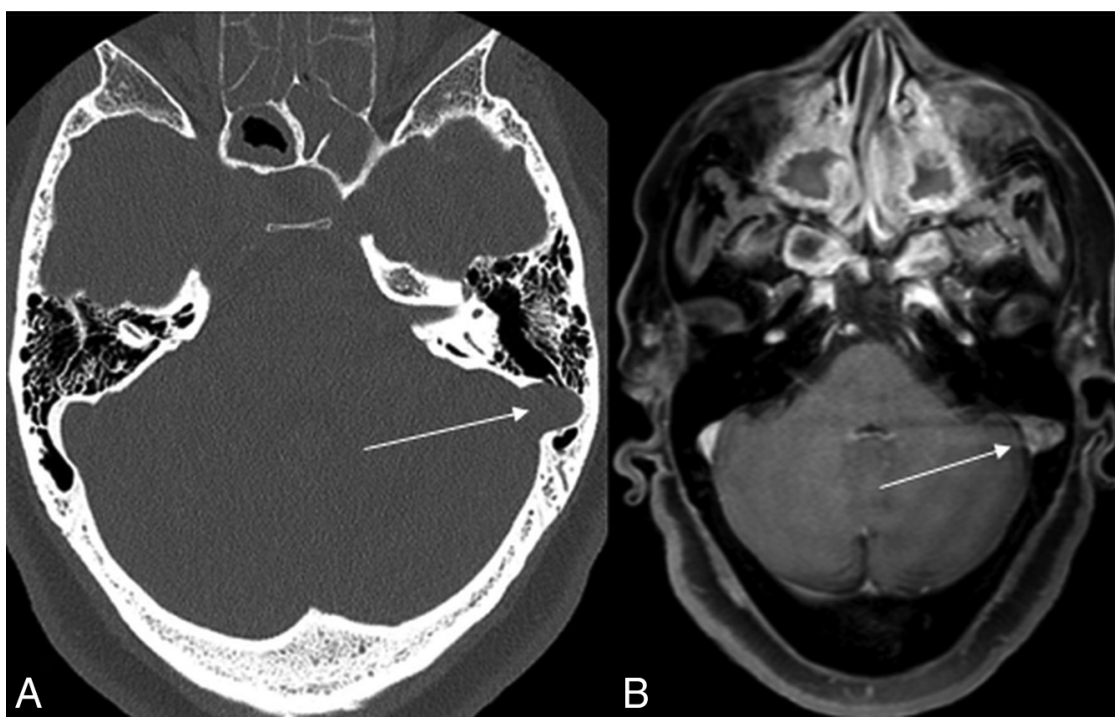

FIG 2. Diverticulum of the left sigmoid sinus (arrow) with focal protrusion of the sigmoid sinus toward the mastoid air cells. The left sigmoid sinus was normal in shape above and below this section ( $A$, Bone window CT; $B$, Postcontrast T1 MR imaging).

sella) was found in 33 patients (58.9\%), while only 4 patients had one through the skull base foramina (ie, the foramen of ovale and the Meckel cave). The remaining patients $(n=19)$ had temporal bone cephaloceles (through the temporal apex and tegmen tympani). In 22 patients (39.3\%), the cephalocele contained brain tissue and meninges (encephalomeningoceles); the remaining 34 patients had cephaloceles that contained only meninges (meningoceles). The average cephalocele volume was $2979 \mathrm{~mm}^{3}$.
The $95 \%$ confidence intervals vary widely due to overfitting of the univariate linear regression $(-3873.20$ to -659.14) supporting a potentially inconclusive finding due to collinearity. To further investigate this inverse association, we performed a univariate logistic regression analysis using the nearest to average volume $\left(3086 \mathrm{~mm}^{3}\right)$ as the cutoff versus the association of SSDD with cephaloceles. An odds ratio of 3.58 of cephaloceles below average volume is associated with SSDD with a $95 \% \mathrm{CI}$, 2.3-4.86 and a $P$ value $=.05$ (Table 2).

\section{DISCUSSION}

To our knowledge, this is the first study to evaluate the prevalence of SSDD in patients with cephaloceles. Within our cohort, the prevalence of SSDD in patients with cephalocele was shown to be $37.5 \%$. We found a strong association between the presence of SSDD and female sex. Similar sex associations have been reported in patients with SSDD in the context of elevated ICPs. ${ }^{11,12}$ In 2017, in the systematic review by Wang et al, ${ }^{17} 90.4 \%$ of patients with pulsatile tinnitus and SSDD were women. ${ }^{17}$ Despite the literature, only 2 of our patients with SSDD had documented evidence of increased ICP and none of our patients presented with pulsatile tinnitus. Our findings match those of a recent study by 


\begin{tabular}{|c|c|c|c|c|}
\hline & Cephalocele Only $(n=35)$ & Cephalocele and SSDD ( $n=21)$ & Total $(n=56)$ & $P$ Value \\
\hline Age (yr) & & & & .71 \\
\hline Mean (SD) & $51.5(13.6)$ & 49.7 (11.9) & $50.8(12.9)$ & \\
\hline Range & $27-79$ & $27-67$ & $27-79$ & \\
\hline Sex & & & & .019 \\
\hline Female & $24(68.6 \%)$ & 20 (95.2\%) & $44(78.6 \%)$ & \\
\hline Male & $11(31.4 \%)$ & $1(4.8 \%)$ & $12(21.4 \%)$ & \\
\hline Symptoms & & & & .235 \\
\hline Bilateral hearing loss & $0(0.0 \%)$ & $1(4.8 \%)$ & $1(1.8 \%)$ & \\
\hline CSF leak & $15(42.9 \%)$ & $9(42.9 \%)$ & $24(42.9 \%)$ & \\
\hline Incidental & $17(48.6 \%)$ & $5(23.8 \%)$ & $22(39.3 \%)$ & \\
\hline Headache secondary to IIH & $1(2.9 \%)$ & $1(4.8 \%)$ & $2(3.6 \%)$ & \\
\hline Meningitis & $1(2.9 \%)$ & $3(14.3 \%)$ & $4(7.1 \%)$ & \\
\hline Meningitis with CSF leak & $1(2.9 \%)$ & $1(4.8 \%)$ & $2(3.6 \%)$ & \\
\hline Otorrhea & $0(0.0 \%)$ & $1(4.8 \%)$ & $1(1.8 \%)$ & \\
\hline Content of cephalocele & & & & .672 \\
\hline Brain and meninges & $13(37.1 \%)$ & $9(42.9 \%)$ & $22(39.3 \%)$ & \\
\hline Meninges & $22(62.9 \%)$ & $12(57.1 \%)$ & $34(60.7 \%)$ & \\
\hline Location & & & & .866 \\
\hline Anterior skull base & $21(60.0 \%)$ & $12(57.1 \%)$ & $33(58.9 \%)$ & \\
\hline Skull base foramina & $2(5.7 \%)$ & $2(9.5 \%)$ & $4(7.1 \%)$ & \\
\hline Temporal bone & $12(34.3 \%)$ & $7(33.3 \%)$ & 19 (33.9\%) & \\
\hline Volume of cephalocele $\left(\mathrm{mm}^{3}\right)$ & & & & .015 \\
\hline Mean (SD) & $3828(3559)$ & 1562 (1511) & 2979 (3144) & \\
\hline Median (minimum-maximum) & $40-12,617$ & $30-4872$ & $30-12,617$ & \\
\hline Cephalocele repaired? & & & & .141 \\
\hline No & $17(48.6 \%)$ & $6(28.6 \%)$ & $23(41.1 \%)$ & \\
\hline Yes & $18(51.4 \%)$ & $15(71.4 \%)$ & $33(58.9 \%)$ & \\
\hline
\end{tabular}

Table 2: Logistic regression for volume less than average $\left(3086 \mathrm{~mm}^{3}\right)$ versus SSDD association with cephalocele

\begin{tabular}{ll}
\hline & Presence of SSDD \\
\hline Volume below average $3086 \mathrm{~mm}^{3}$ & $\mathrm{OR}=3.58$ \\
& $95 \% \mathrm{Cl}, 2.30-4.86$ \\
& $P=.05$ \\
Constant & 0.25 \\
& $(-0.85-1.35)$ \\
Observations & 56 \\
Log likelihood & -34.91 \\
Akaike information criterion & 73.81 \\
\hline
\end{tabular}

Lansley et $\mathrm{al}^{16}$ and raise questions with regard to SSDD as the proximal cause of pulsatile tinnitus. In patients with IIH, the pulsatile tinnitus can be secondary to IIH itself rather than SSDD.

The inverse relationship noted between the volume of cephalocele and SSDD will require further investigation. One potential explanation is that increased ICP may manifest in several different ways (eg, empty sella, prominent subarachnoid spaces around optic nerves, cephalocele formation, and SSDD). Accordingly, one may postulate that once SSDD develops in patients with cephaloceles, this anomaly provides a "pressure outlet" so that elevated ICP is more likely to contribute to further dehiscence in lieu of continued cephalocele growth. Also, an association between SSDD and cephaloceles may favor CSF pulsation as the cause of the SSDD as opposed to venous pulsations.

A little less than half of our patients were found to have CSF rhinorrhea that required treatment, and in our cohort, the second most common reason for discovery of the cephalocele was purely incidental. Current treatment options for symptomatic SSDD include the classic transmastoid and retrosigmoid open surgical approaches; more recent endovascular approaches involving coil embolization and stent placement have also begun to emerge. ${ }^{12-14,18}$ Given our findings and recent literature, we recommend evaluation of IIH before invasive treatment of SSDD in patients with pulsatile tinnitus.

\section{Limitations}

Our study has a few limitations. First, it is a retrospective analysis that includes a relatively heterogeneous group of patients and imaging from our institutional data base; thus, it does not include patients who may have received treatment at outside institutions. Second, only the sigmoid sinus was evaluated, and the presence of additional intracranial venous anomalies and stenosis could not be evaluated, given that dedicated CT MR venography of both were not performed. Third, this is a relatively small cohort that may not be adequately powered to find subtle differences between groups with and without SSDD. Statistically, the study is underpowered and does not offer conclusive evidence for its findings but offers the first literature reports of possible associations among sex, the presence of SSDD, and its volume with cephaloceles. While our study found a significant inverse relationship between cephalocele volume and the presence of SSDD $(P=.036)$, the lack of a strong linear association (per $R^{2}=0.1077$ ) may ultimately reflect an underpowered study. Our logistic regression demonstrated an odds ratio of 3.58 for smaller volumes and increased association with SSDD with a $P$ value at exactly .05 while using the cutoff of average volume, though the range of volumes for the cephaloceles analyzed is wide. A more extensive investigation is warranted and may ultimately be accomplished via the provision of an appropriately powered multi-institutional study. 


\section{CONCLUSIONS}

Within our cohort, the prevalence of SSDD in patients with a cephalocele was shown to be $37.5 \%$. Herein we report a potential association between female sex and an inverse relationship between cephalocele volume and SSDD. We did not find any differences between the presence and absence of SSDD in patients with cephaloceles when comparing age, location of cephalocele, content, or clinical symptoms. SSDD and skull base cephaloceles may share an underlying common pathophysiology such as IIH. Further studies are needed to evaluate the exact mechanisms between these skull base and venous pathologies.

Disclosures: Joshua D. Bernstock-RELATED: positions and equity in CITC Ltd and Avidea Technologies and a member of the POCKiT Diagnostic Board of Scientific Advisors.

\section{REFERENCES}

1. Connor SE. Imaging of skull-base cephalocoeles and cerebrospinal fluid leaks. Clin Radiol 2010;65:832-41 CrossRef Medline

2. Lloyd KM, DelGaudio JM, Hudgins PA. Imaging of skull base cerebrospinal fluid leaks in adults. Radiology 2008;248:725-36 CrossRef Medline

3. Naidich TP, Altman NR, Braffman BH, et al. Cephaloceles and related malformations. AJNR Am J Neuroradiol 1992;13:655-90 Medline

4. Mann SS, Naidich TP, Towbin RB, et al. Imaging of postnatal maturation of the skull base. Neuroimaging Clin N Am 2000;10:1-21 Medline

5. Wilkins RH, Radtke RA, Burger PC. Spontaneous temporal encephalocele: case report. J Neurosurg 1993;78:492-98 CrossRef Medline

6. Gacek RR, Gacek MR, Tart R. Adult spontaneous cerebrospinal fluid otorrhea: diagnosis and management. Am J Otol 1999;20:770-76 Medline

7. Shetty PG, Shroff MM, Fatterpekar GM, et al. A retrospective analysis of spontaneous sphenoid sinus fistula: MR and CT findings. AJNR Am J Neuroradiol 2000;21:337-42 Medline
8. Chang DW, Langstein HN, Gupta A, et al. Reconstructive management of cranial base defects after tumor ablation. Plast Reconstr Surg 2001;107:1346-55; discussion 56-57 CrossRef Medline

9. Yilmazlar S, Arslan E, Kocaeli H, et al. Cerebrospinal fluid leakage complicating skull base fractures: analysis of 81 cases. Neurosurg Rev 2006;29:64-71 CrossRef Medline

10. Harvey RS, Hertzano R, Kelman SE, et al. Pulse-synchronous tinnitus and sigmoid sinus wall anomalies: descriptive epidemiology and the idiopathic intracranial hypertension patient population. Otol Neurotol 2014;35:7-15 CrossRef Medline

11. Liu Z, Chen C, Wang Z, et al. Sigmoid sinus diverticulum and pulsatile tinnitus: analysis of CT scans from 15 cases. Acta Radiol 2013;54:81286 CrossRef Medline

12. Schoeff S, Nicholas B, Mukherjee S, et al. Imaging prevalence of sigmoid sinus dehiscence among patients with and without pulsatile tinnitus. Otolaryngol Head Neck Surg 2014;150:841-46 CrossRef Medline

13. Eisenman DJ. Sinus wall reconstruction for sigmoid sinus diverticulum and dehiscence: a standardized surgical procedure for a range of radiographic findings. Otol Neurotol 2011;32:1116-19 CrossRef Medline

14. Otto KJ, Hudgins PA, Abdelkafy W, et al. Sigmoid sinus diverticulum: a new surgical approach to the correction of pulsatile tinnitus. Otol Neurotol 2007;28:48-53 CrossRef Medline

15. Hou ZQ, Han DY. Pulsatile tinnitus in perimenopausal period. Acta Otolaryngol 2011;131:896-904 CrossRef Medline

16. Lansley JA, Tucker W, Eriksen MR, et al. Sigmoid sinus diverticulum, dehiscence, and venous sinus stenosis: potential causes of pulsatile tinnitus in patients with idiopathic intracranial hypertension? AJNR Am J Neuroradiol 2017;38:1783-88 CrossRef Medline

17. Wang AC, Nelson AN, Pino C, et al. Management of sigmoid sinus associated pulsatile tinnitus: a systematic review of the literature. Otol Neurotol 2017;38:1390-96 CrossRef Medline

18. Shastri RK, Chaudhary N, Pandey AS, et al. Venous diverticula causing pulsatile tinnitus treated with coil embolization and stent placement with resolution of symptoms: report of two cases and review of the literature. Otol Neurotol 2017;38:e302-07 CrossRef Medline 